\title{
Thermography assessment of spastic lower limb in patients after cerebral stroke undergoing rehabilitation
}

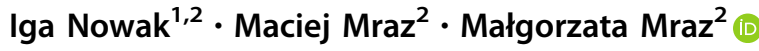

Received: 30 April 2019/Accepted: 15 September 2019/Published online: 26 September 2019

(C) The Author(s) 2019

\begin{abstract}
Stroke contributes to disability in adulthood. The assessment of the degree of spasticity is one of the basic methods of patient examination after stroke and is used for monitoring rehabilitative outcomes; however, no optimal scale for the unambiguous assessment of spasticity exists. Our study aimed to assess the usefulness of thermography in measuring the effects of rehabilitation in stroke patients' spastic hemiparesis. In this prospective, single-center study, 40 stroke patients with a mean age of $60.6 \pm 5.5$ years were enrolled. All suffered from hemiparesis. Surface temperature of the shank was assessed with infrared thermography; degree of independence with the Barthel Index; and muscle tone with the Modified Ashworth Scale. A comparison of temperature between the spastic and non-spastic posterior part of the shank was conducted. Temperature changes, severity of spasticity, and gait pattern were evaluated and compared at baseline and after 6 weeks of rehabilitation. All patients completed a rehabilitation program. The baseline temperature in the spastic extremity was significantly lower than in the normal extremity (mean temperature $28.93{ }^{\circ} \mathrm{C}$ vs $30.20{ }^{\circ} \mathrm{C} ; p=0.0001$ ). After rehabilitation, the temperature in the spastic extremity increased significantly; however, the significant difference between the two extremities persisted (mean temperature $29.76{ }^{\circ} \mathrm{C}$ vs $30.54{ }^{\circ} \mathrm{C} ; p=0.0001$ ). After rehabilitation, spasticity in affected extremity decreased significantly $(p>0.001)$. Additionally, an improvement in gait pattern was observed. We conclude that thermography proved to be useful in the assessment of rehabilitation effects in stroke patients with spastic hemiparesis; thus, it can be considered an additional tool for determining impaired muscle tone in patients with spasticity.
\end{abstract}

Keywords Spasticity · Gait · Stroke · Rehabilitation · Thermography · Thermovision

\section{Introduction}

Stroke is one of the major causes of disability in adulthood. Regaining independence after stroke may be difficult due to spasticity in the paralyzed extremities [1]. Pathologically increased muscle tone remains one of the primary causes of intentional movement impairment and dyskinesia, including gait disorders [2, 3]. In patients after stroke, the pathological gait is caused by a decrease in muscle

Małgorzata Mraz

malgorzata.mraz@awf.wroc.pl

1 Specialist Centre of Prophylaxis and Treatment "PROVITA", ul. Bierutowska 63, 51-317 Wrocław, Poland

2 Faculty of Physiotherapy, University of Physical Education in Wroclaw, Aleja Ignacego Jana Paderewskiego 35, 51-612 Wrocław, Poland strength, neuromuscular control disorders, spasticity, and pain; secondary to these, the limitation of mobility in joints as well as foot deformation translates into disorders in all phases of gait (Fig. 1a, b) [4-6]. Reduced strength and spasticity affect thermoregulation, and for this reason, thermography can be considered as a useful tool for the assessment of spasticity in stroke patients. Proper diagnosis of gait disorders in stroke patients offers an opportunity to set specific goals for rehabilitation that should significantly contribute to a decrease in spasticity and improvement in functioning, in terms of gait [1].

Physiotherapy and its techniques and methods of neurorehabilitation are an integral element of treatment of spasticity and its consequences. There is no one strictly defined physiotherapy model for patients after stroke. However, there are many studies which confirm the efficacy of functional therapy $[7,8]$. 
Fig. 1 Spastic positioning of the right lower limb during transfer (a back view; b front view)
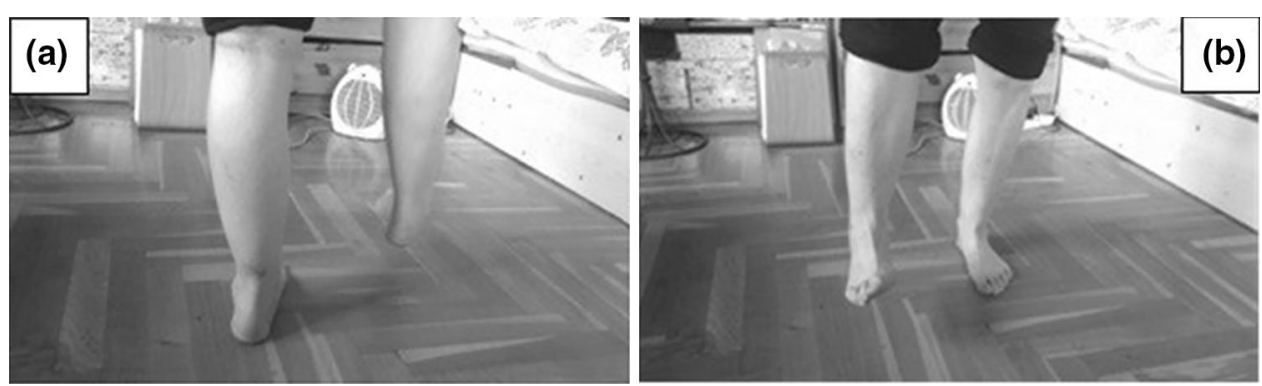

The assessment of the degree of spasticity is one of the basic methods of patient examination after stroke and is used for monitoring rehabilitative outcomes [9]. Clinical methods measure positive and negative symptoms associated with increased muscle tone, such as tendon reflexes; clonuses and muscle spasms; muscle strength; range of movement in joints; and ability and purposefulness of intentional movement [10]. There is no, however, any optimal scale for the unambiguous assessment of spasticity, as it is not a physical measure. In clinical conditions, the Modified Ashworth Scale (MAS) is often applied [11-13], but clinical assessment methods of spasticity have proven to be of low reliability and lack objectivity [13]. For this reason, they should be used in tandem with other functional scales.

Many pathological processes in the human body can change temperature mostly due to changes in vascular flow. This in turn affects the temperature of adjacent tissues, including the skin. Hence, there is a possibility of using thermography in assessing the value of those changes and their spatial distribution [14]. Reports from the literature on using thermography in evaluation of benefits of rehabilitation are rare. Alfieri et al. [15] showed that paretic side of patients after stroke had lower temperature than respective side in healthy volunteers. The work by Hegedus confirmed the potential of thermography to monitor improvement after rehabilitation. The study showed a significant increase in temperature of the affected extremities [16]. Differences in temperature were linked to microcirculatory disorders in the affected extremities. Thermography examination is noninvasive and totally safe for patients. It is based on the measurement of infrared radiation emitted by the part of the surface of the body being studied [17]. Thermography is becoming more widely used in physiotherapy including recent attempt to evaluate rehabilitation benefits in stroke patients $[15,16]$.

\section{Aim of the study}

The aim of the study was to assess the usefulness of thermography in measuring the effects of rehabilitation in stroke patients with spastic hemiparesis.
Research questions:

1. Is there a difference between the thermography image of a lower limb affected by spastic paresis and the thermography image of the healthy limb (the back of the shank)?

2. Does the degree of spasticity have any influence on the surface temperature of the back region of the calf?

3. Is thermography useful for monitoring rehabilitation in patients after cerebral stroke?

\section{Materials and methods}

The study included 40 patients (15 men and 25 women) after ischemic and hemorrhagic stroke presenting spasticity within the gastrocnemius muscle and/or the soleus muscle. The time since the event was from 3 to 12 months. All patients gave their informed consent for participation in the study. The study was approved by the Senate Committee for Research Ethics at University of Physical Education in Wrocław, Poland. This ethics committee is legally entitled to give permission for performing research on humans conducted at the University of Physical Education in Wroclaw, Poland. The study was conducted in accordance with the Declaration of Helsinki.

For the thermographic assessment of lower limbs, the thermal imaging camera FLIR T335 (FLIR Systems, Wilsonville, OR, USA) was used. Curves were analyzed using ThermaCAM Researcher Pro 2.10 software. The device had the following parameters:

- Temperature measurement range: from -20 to $650{ }^{\circ} \mathrm{C}$ ( -4 to $\left.1202{ }^{\circ} \mathrm{F}\right)$;

- Thermal sensitivity (NETD): $<0.05{ }^{\circ} \mathrm{C}$ in ambient temperature up to $30{ }^{\circ} \mathrm{C}$;

- Field of view: $25^{\circ} \times 19^{\circ} / 1.31 \mathrm{ft}(0.4 \mathrm{~m})$;

- Detector type: uncooled microbolometer $320 \times 240$ pixels;

- Image modes: IR image/visual image/picture in picture (merged thermo- and photograms);

- Spectral range: 7.5-13 $\mu \mathrm{m}$; 
- Emissivity value was set to default value determined by the producer equal to 0.98 .

Curves of the shank were performed twice: before rehabilitation and after 6 weeks of rehabilitation. Patients were instructed not to participate in any physical treatments and to avoid physical effort before the examination, to avoid using stimulants, large meals, cosmetics, exposure to sun, and showering before examination. The thermography camera was located at a $2-\mathrm{m}$ distance from the patient, at the height of $1 \mathrm{~m}$. Thermal imaging examinations were conducted in a room of $20 \mathrm{~m}^{2}$ adapted for use, under constant microclimatic conditions $22-23{ }^{\circ} \mathrm{C}, 55 \%$ humidity, without heat radiators or rapid airflow. Those parameters were kept constant for each patient [17, 18]. The average temperature during the examinations was $22 \pm 0.5^{\circ} \mathrm{C}$.

On each curve, the region of interest (ROI) was analyzed. The ROI was designated by a rectangle created by two lines: one passing through the narrowest point between the medial and lateral malleolus and the second passing through the narrowest point below the knee joint. Examples of curves are presented in Fig. 2a, b. Symmetric rectangles of equal surface area were determined on both shanks (healthy and affected by paresis) [19]. A program for the analysis of curves determined the minimum, maximum, and average values of the measured temperature. During each examination, three curves of the back side of the shank were performed and used to calculate the mean value.

For the assessment of muscle tone, MAS was used. This scale was developed to measure resistance during passive soft-tissue stretching. The examination was performed twice: before rehabilitation and after 6-week rehabilitation. During the muscle tone assessment, the patient lay in a supine position with a plantar-flexed ankle and the hip in neutral rotation and flexion. Next, a passive movementthe dorsiflexion of the ankle from the maximum possible plantar flexion to the maximum possible dorsiflexion-was performed by a physician to rate the muscle tone of gastrocnemius muscle [20]. At the same visit, the Barthel
Index Scoring Form for establishing the degree of independence was completed by a physician based on the patient's and relatives' answers as well as direct observation of the patient [21].

Rehabilitation after cerebral stroke was conducted for 6 weeks in an inpatient setting at the Department of Neurological Rehabilitation. Physiotherapeutic treatments were conducted 5 days a week in two cycles, in the morning and in the afternoon. On the sixth day, patients strengthened acquired skills and participated in one cycle of the program. They performed exercises regulating muscle tone; mobilization exercises of the smaller and large joints; reeducation exercises for gait; and balance exercises. The therapy was conducted using the following methods: neurodevelopmental treatment-Bobath (NDT-Bobath), proprioceptive neuromuscular facilitation (PNF), kinesiology taping, and manual therapies. An important part of the therapy to decrease spasticity was applying proper positioning to prevent pathologic synergistic limb and trunk patterns.

The results of the study were collected in an Excel spreadsheet and analyzed with Statistica software PL v.12 (StatSoft, Tulsa, OK, USA). First, the Kolmogorov-Smirnov test was used to test for normal distribution. It was possible to analyze men and women together as sex did not significantly differentiate the study group. The degree of spasticity before and after rehabilitation was compared using the Wilcoxon signed-rank test. The analysis of thermography results of the temperature measurements between the spastic and healthy lower limb was conducted using analysis of variance and the post hoc Duncan's test. A probability value of $p<0.05$ was considered statistically significant.

\section{Results}

All 40 stroke patients completed the study and were analyzed. The age of the study group ranged from 47 to 70 years $(60.6 \pm 5.5)$. Of the study group, 19 patients
Fig. 2 A patient with a spastic left limb (a before the treatment; $\mathbf{b}$ after the treatment)
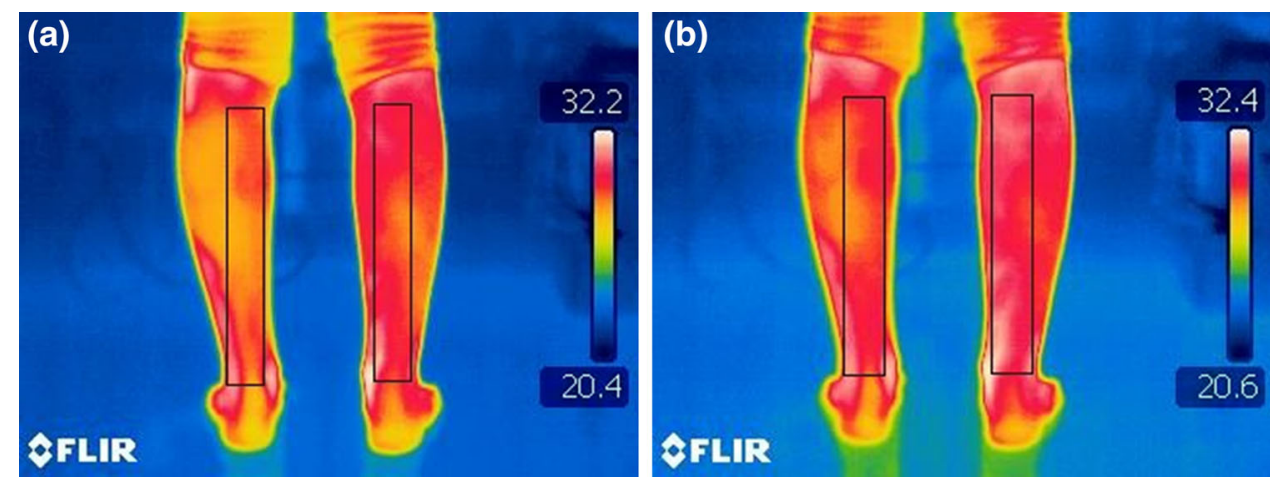
(eight men, 11 women) had right-sided hemiparesis, while 21 (seven men, 14 women) had left-sided hemiparesis. The time since diagnosis ranged from 3 to 10 months $(5.05 \pm 2.07)$. The severity of spasticity in the lower limb ranged from grade 2 to 3 on MAS (2.5 \pm 0.5$)$ : 19 patients (14 men, five women) presented with spasticity of grade 2 and 21 (11 men, ten women) with grade 3 . Independence in daily activities according to Barthel Index ranged from 40 to $60(54.13 \pm 5.87)$. Patients' characteristics are presented in Table 1.

The comparison of temperature between the spastic and normal extremity revealed significant differences. At baseline, the thermography assessment of the spastic extremity was characterized by significantly lower minimum, maximum, and average temperatures than those in the normal extremity (Table 2, Fig. 3). After 6-week rehabilitation, the significant difference between spastic and normal extremity persisted (Table 3); however, a significant increase in minimal $(p>0.001)$, maximal $(p>0.001)$, and average $(p>0.001)$ temperature in the spastic extremity was observed (Fig. 4).

After rehabilitation, spasticity in the affected extremity decreased significantly $(p>0.001)$. One patient presented with grade 3 spasticity, 18 patients with grade 2, eight patients with grade 1.5 , and 13 patients with grade 1 (Fig. 3). Spasticity decreased in each subsequent week of rehabilitation, which translated into improvement in patients' gait patterns. Additionally, a decrease in spasticity as assessed with MAS was associated with an increase in surface temperature in the assessed region of the spastic extremity.

\section{Discussion}

In this study, we evaluated the usefulness of thermography in measuring the effects of rehabilitation in stroke patients with spastic hemiparesis. In patients after cerebral stroke, a 6-week hospital rehabilitation resulted in a decrease in spasticity in the lower extremity. Along with a decrease in spasticity, an increase in the surface temperature in the back side of the spastic shank was observed. Thermographic examination proved to be useful in the assessment of the severity of spasticity as well as in the objective monitoring of pathological muscle tone in patients after cerebral stroke undergoing physiotherapy treatment.

Spastic paresis of a lower extremity, as suggested by other researchers, may result not only in gait disorders and difficulties in mobility, but may worsen levels of physical activity and functional ability [2, 3]. The aim of the study performed by Tao et al. was to establish whether a lowdose botulinum toxin A injected into the muscles of the shank in patients after cerebral stroke would improve gait and daily living activities by decreasing spasticity [22]. The control group from that study received a placebo. The study showed a significant relationship between the decrease in spasticity as measured with MAS and the level of gait improvement and functional independence with the reduced activation of muscles.

Francis et al. concluded that a reduction in spasticity translated into an increase in physical activity [1, 23]. Besides the relationship between changes in the degree of spasticity and function, the authors showed the significant influence of time, which can be explained by the need for the central nervous system and the structures of the unaffected extremity to reorganize themselves. Results obtained in the present study revealed gradual functional improvement in patients undergoing 6-week rehabilitation targeted at lowering spasticity. Monitoring the effects of rehabilitative management showed an improvement in patient functioning from the third week of physiotherapy. Increasingly better functional status was achieved until the last week of the therapy. In the present study, greater gait impairment was observed in patients with MAS grade 3 spasticity than in patients with less severe spasticity (MAS grade 2). A similar relationship was found in the impairment of physical activity in patients with greater spasticity; daily functioning was significantly worse for them in those with lesser spasticity.

Many researchers report that the outcomes of conducted therapy are independent of age and sex [24-26]. The results of the present study confirm this observation. Statistical analysis revealed that variables such as sex and age did not significantly differentiate the study group.

The present study attempted to answer the question of whether thermography was useful in assessing changes in the severity of spasticity in patients with hemiparesis after cerebral stroke. A significant change in the increased muscle tone was observed as an effect of the applied
Table 1 Characteristics of the study group

\begin{tabular}{lcccc}
\hline & Average Value (SD) & Median & Range & IQR \\
\hline Age; years & $60.60(5.50)$ & 61 & $46-70$ & $58-64$ \\
Months after stroke & $5.05(2.07)$ & 4 & $3-10$ & $3-7$ \\
Barthel Index & $54.13(5.87)$ & 55 & $40-60$ & $50-60$ \\
Modified Ashworth Scale & $2.53(0.51)$ & 3 & $2-3$ & $2-3$ \\
\hline
\end{tabular}


Table 2 Post hoc comparison of surface temperature between spastic and normal shank at baseline

\begin{tabular}{|c|c|c|c|c|c|c|c|}
\hline $\begin{array}{l}d f=78 \\
\mathrm{SE}=0.3974\end{array}$ & Average value & MIN spastic & MIN normal & MAX spastic & MAX normal & AVG spastic & AVR normal \\
\hline MIN spastic & 29.95 & & 0.0001 & $<0.0001$ & 0.0001 & 0.0001 & 0.0743 \\
\hline MIN normal & 31.44 & 0.0001 & & $<0.0001$ & $<0.0001$ & $<0.0001$ & 0.0001 \\
\hline MAX spastic & 26.66 & $<0.0001$ & $<0.0001$ & & 0.0001 & 0.0001 & $<0.0001$ \\
\hline MAX normal & 28.40 & 0.0001 & $<0.0001$ & 0.0001 & & 0.0004 & $<0.0001$ \\
\hline AVG spastic & 28.93 & 0.0001 & $<0.0001$ & 0.0001 & 0.0004 & & 0.0001 \\
\hline AVR normal & 30.20 & 0.0743 & 0.0001 & $<0.0001$ & $<0.0001$ & 0.0001 & \\
\hline
\end{tabular}

AVR, average surface temperature; MAX, maximum surface temperature; MIN, minimum surface temperature; values in bold denote statistical significance
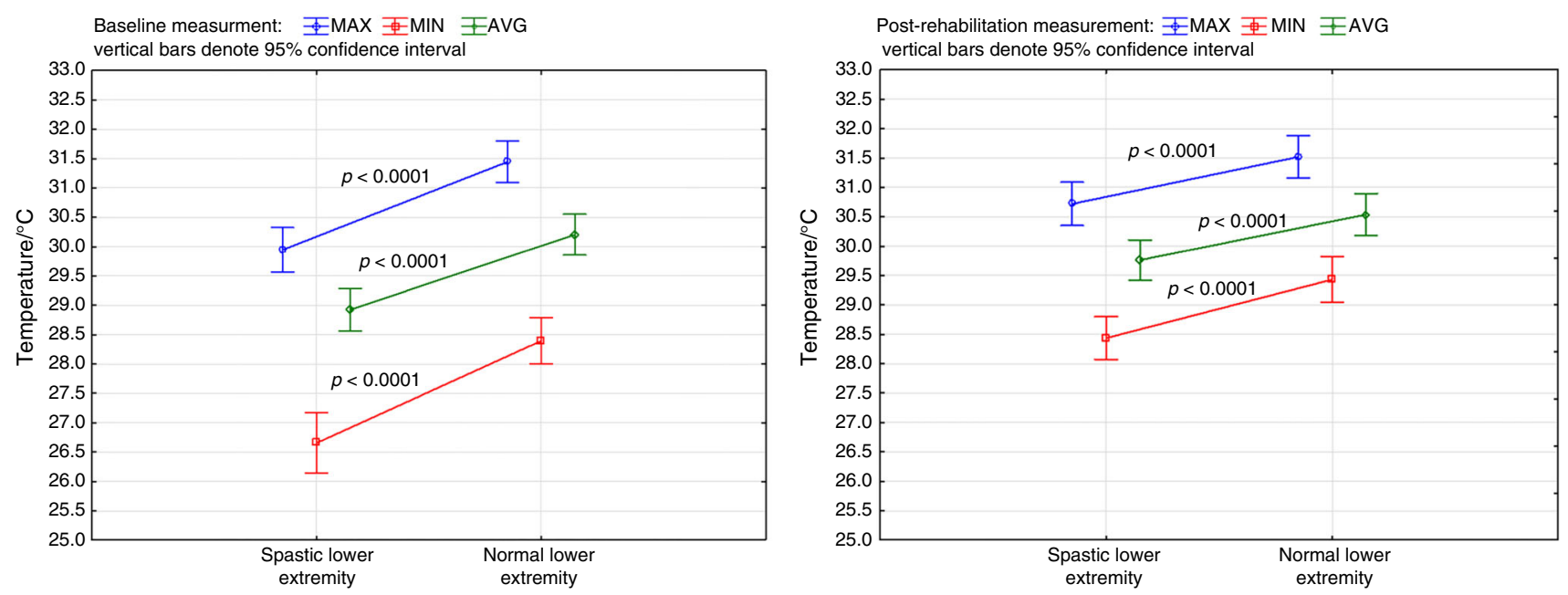

Fig. 3 Comparison of surface temperature between spastic and normal shank at baseline and at 6 weeks of rehabilitation

Table 3 Post hoc comparison of surface temperature between spastic and normal shank after 6-week rehabilitation

\begin{tabular}{|c|c|c|c|c|c|c|c|}
\hline $\begin{array}{l}d f=78 \\
\mathrm{SE}=0.0767\end{array}$ & Average value & MIN spastic & MIN normal & MAX spastic & MAX normal & AVG spastic & AVR normal \\
\hline MIN spastic & 30.72 & & 0.0001 & $<0.0001$ & 0.0001 & 0.0001 & 0.0049 \\
\hline MIN normal & 31.52 & 0.0001 & & $<0.0001$ & $<0.0001$ & $<0.0001$ & 0.0001 \\
\hline MAX spastic & 28.44 & $<0.0001$ & $<0.0001$ & & 0.0001 & 0.0001 & $<0.0001$ \\
\hline MAX normal & 29.43 & $<0.0001$ & $<0.0001$ & 0.0001 & & 0.0001 & 0.0001 \\
\hline AVG spastic & 29.76 & 0.0001 & $<0.0001$ & 0.0001 & 0.0001 & & 0.0001 \\
\hline AVR normal & 30.54 & 0.0049 & 0.0001 & $<0.0001$ & $<0.0001$ & 0.0001 & \\
\hline
\end{tabular}

AVR, average surface temperature; MAX, maximum surface temperature; MIN, minimum surface temperature; values in bold denote statistical significance

physiotherapy treatment. In patients undergoing physiotherapy, spasticity decreased as measured with MAS. The assessment of spasticity revealed functional improvement in gait patterns and better functional activity as measured on subjective scales. Functional improvement can be visible on thermography possibly due to improvement in thermoregulatory processes following better blood flow and heat production as it has been shown that exercises improve efficiency of thermoregulatory processes [27, 28].

The results of thermography were interesting, as well. The present study shows significant differences in the surface temperatures of the shank region in the spastic 
Fig. 4 Comparison of the severity of spasticity in the paretic extremity at baseline and after rehabilitation
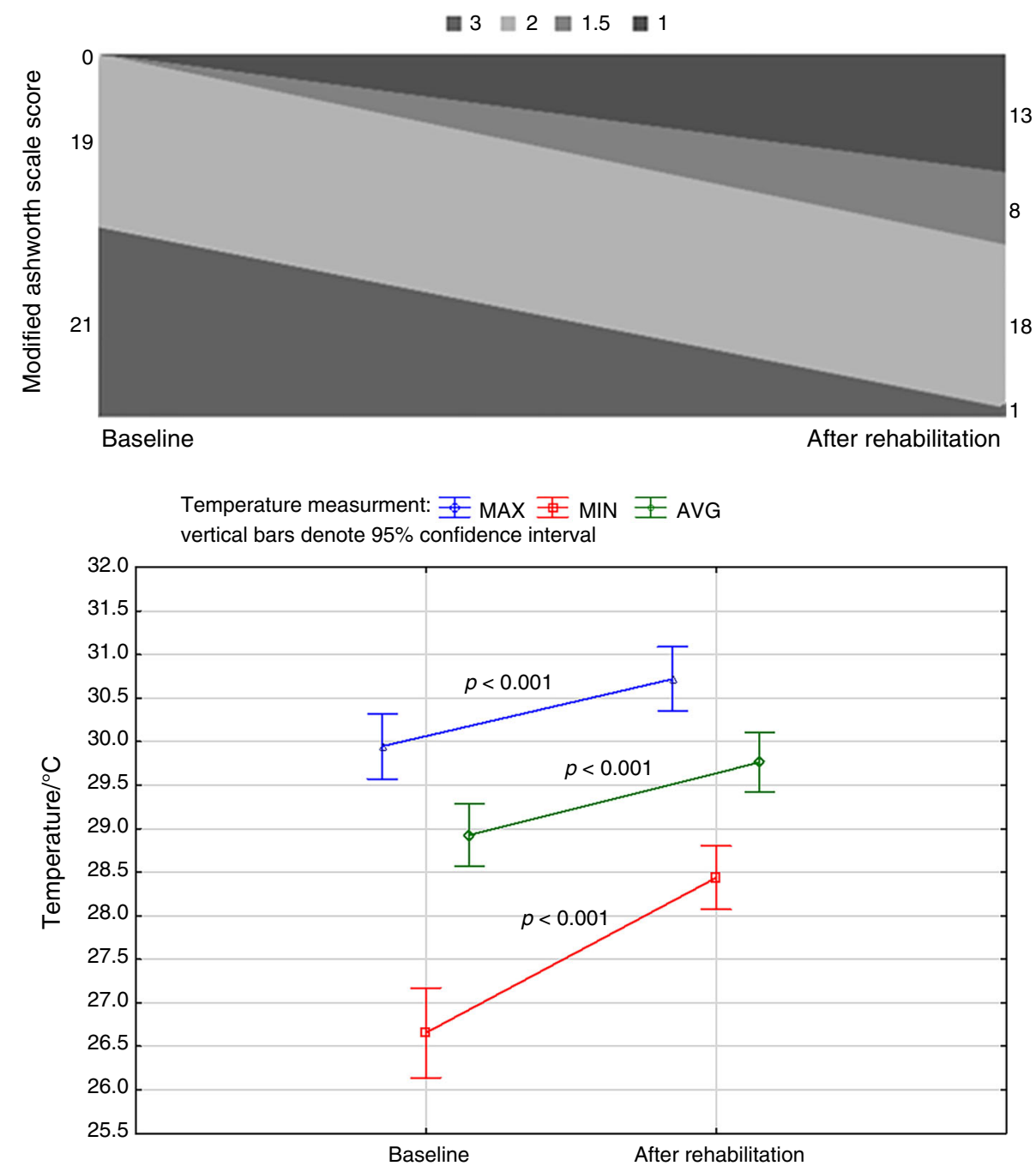

extremity and the normal one. At baseline, the surface temperature of the spastic extremity was on average $1.5^{\circ} \mathrm{C}$ lower than that recorded on the surface of the unaffected leg (Fig. 3). Throughout the rehabilitative procedure and the resultant decrease in spasticity, this difference diminished significantly (Fig. 4) due to improvement in thermoregulatory and physiological processes. A comparison of thermal images and conducted statistical analysis of the surface temperature results revealed an increase in surface temperature of the spastic limb. Along with the decrease in spasticity in the lower spastic extremity, an increase in the surface temperature of the areas assessed with thermography was observed.

Thermography based on thermal emission of tissues of the human body creates great diagnostic capabilities. Homeothermy is associated with metabolism, and its physiologic changes may be recorded by thermography; however, areas that present with different temperature distribution than normal tissues may be affected by pathological processes. Christensen et al. used thermography to evaluate postoperative inflammation after the removal of the mandibular third molar. On the second day post-surgery, the operated side of the face was significantly warmer by $0.33{ }^{\circ} \mathrm{C}$ than the control [29]. Bagavathiappan et al. [30] reported a significant difference in mean foot surface temperature between diabetic patients with neuropathy and without. Neuropathic feet were warmer by $1.33{ }^{\circ} \mathrm{C}$. Lim et al. proved that thermography can be used in the diagnosis of Raynaud's phenomenon. If the temperature difference between the dorsum of the feet and the first toe exceeds $3.11{ }^{\circ} \mathrm{C}$ or the temperature difference between the palm of the hand and the coolest finger exceeds $2.2^{\circ} \mathrm{C}$ in men, it is highly suggestive of the presence of Raynaud's phenomenon [31]. The temperature difference between the normal and affected area depends on the type of pathology. In the present study, the difference between the healthy and spastic shank was $1.5^{\circ} \mathrm{C}$. However, it dropped after rehabilitation to $0.8{ }^{\circ} \mathrm{C}$, indicating improvement in the condition of the treated extremity. 
Reports on the use of thermography for monitoring improvement after rehabilitation in stroke patients with paresis are scarce. Dymarek et al. monitored the effects of extracorporeal shock wave application for wrist and finger spasticity. They reported a significant reduction in MAS and muscle tone as well as an increase in surface temperature. The surface temperature was significantly higher by $1.35{ }^{\circ} \mathrm{C}$ at $24 \mathrm{~h}$ after extracorporeal shock wave treatment than at baseline $\left(33.48 \pm 2.07{ }^{\circ} \mathrm{C}\right.$ vs $\left.32.13 \pm 2.26{ }^{\circ} \mathrm{C}\right)$ [32]. The results obtained by Dymarek et al. cannot be compared directly with findings from the present study because of the different therapies used and different observation times, but it is notable that an improvement in condition of spastic extremity was associated with a rise in surface temperature, which is in line with our result.

\section{Limitations}

The study included homogeneous group of stoke patients with hemiparesis and spasticity within the gastrocnemius and/or soleus muscle undergoing rehabilitation 3 to 12 month postevent. Due to using strict inclusion criteria, the results can be applied only to this specific patients' group. However, thermography proved to be useful in the assessment of the severity of spasticity as well as in the objective monitoring of pathological muscle tone in patients after cerebral stroke undergoing physiotherapy treatment, and the results cannot be transferred directly into clinical practice. This method needs further studies to determine cutoff levels and to check whether it can be used alone. Nevertheless, our study presents promising results and the potential of using thermography at rehabilitation wards, especially because of its simplicity, low costs, and capacity of giving reproducible results.

\section{Conclusions}

Thermography proved to be useful in the assessment of the effects of rehabilitation in stroke patients with spastic hemiparesis. After 6-week rehabilitation, an increase in surface temperature was accompanied by a decrease in spasticity and improvement in gait pattern. Thermography can be considered an additional tool for determining impaired muscle tone in patients with spasticity.

Funding No funding was received for this work.

\section{Compliance with ethical standards}

Conflict of interest The authors declare that they have no conflict of interest.
Open Access This article is distributed under the terms of the Creative Commons Attribution 4.0 International License (http://creative commons.org/licenses/by/4.0/), which permits unrestricted use, distribution, and reproduction in any medium, provided you give appropriate credit to the original author(s) and the source, provide a link to the Creative Commons license, and indicate if changes were made.

\section{References}

1. Francis HP, Wade DT, Turner-Stokes L, Kingswell RS, Dott CS, Coxon EA. Does reducing spasticity translate into functional benefit? An exploratory meta-analysis. J Neurol Neurosurg Psychiatry. 2004;75(11):1547-51.

2. Patterson SL, Forrester LW, Rodgers MM, et al. Determinants of walking function after stroke: differences by deficit severity. Arch Phys Med Rehabil. 2007;88(1):115-9.

3. Siniscalchi A, Gallelli L, Labate A, Malferrari G, Palleria C, Sarro GD. Post-stroke movement disorders: clinical manifestations and pharmacological management. Curr Neuropharmacol. 2012;10(3):254-62.

4. Sullivan KJ, Brown DA, Klassen T, et al. Effects of task-specific locomotor and strength training in adults who were ambulatory after stroke: results of the STEPS randomized clinical trial. Phys Ther. 2007;87(12):1580-602.

5. Manca M, Marchi P. A proposal of clinical report regarding dynamic Emg in gait disorders following upper motor neuron lesion. Open Rehabil J. 2010;3:151-7.

6. Yan T, Hui-Chan CW, Li LS. Functional electrical stimulation improves motor recovery of the lower extremity and walking ability of subjects with first acute stroke: a randomized placebocontrolled trial. Stroke. 2005;36(1):80-5.

7. Pollock A, Farmer SE, Brady MC, et al. Interventions for improving upper limb function after stroke. Cochrane Database Syst Rev. 2014. https://doi.org/10.1002/14651858.CD010820. pub2.

8. Van Peppen RP, Kwakkel G, Wood-Dauphinee S, Hendriks HJ, Van der Wees PJ, Dekker J. The impact of physical therapy on functional outcomes after stroke: what's the evidence? Clin Rehabil. 2004;18(8):833-62.

9. Aloraini SM, Gaverth J, Yeung E, MacKay-Lyons M. Assessment of spasticity after stroke using clinical measures: a systematic review. Disabil Rehabil. 2015;37(25):2313-23.

10. Lebiedowska MK, Fisk JR. Quantitative evaluation of reflex and voluntary activity in children with spasticity. Arch Phys Med Rehabil. 2003;84(6):828-37.

11. Voerman GE, Gregoric M, Hermens HJ. Neurophysiological methods for the assessment of spasticity: the Hoffmann reflex, the tendon reflex, and the stretch reflex. Disabil Rehabil. 2005;27(1-2):33-68.

12. Burridge JH, Wood DE, Hermens HJ, et al. Theoretical and methodological considerations in the measurement of spasticity. Disabil Rehabil. 2005;27(1-2):69-80.

13. Pandyan AD, Gregoric M, Barnes MP, et al. Spasticity: clinical perceptions, neurological realities and meaningful measurement. Disabil Rehabil. 2005;27(1-2):2-6.

14. Ring EF. The historical development of thermometry and thermal imaging in medicine. J Med Eng Technol. 2006;30(4):192-8.

15. Alfieri FM, Massaro AR, Filippo TR, Portes LA, Battistella LR. Evaluation of body temperature in individuals with stroke. Neurorehabilitation. 2017;40(1):119-28.

16. Hegedus B. The potential role of thermography in determining the efficacy of stroke rehabilitation. J Stroke Cerebrovasc Dis. 2018;27(2):309-14. 
17. Ring E, Ammer K, Jung A, et al. Standardization of infrared imaging. In: The 26th Annual International Conference of the IEEE Engineering in Medicine and Biology Society, vol. 2, pp 1183-5. 2004.

18. Clark R, de Calcina-Goff $M$. International standardization in medical thermography. In: Proceedings of 18th Annual International Conference of the IEEE Engineering in Medicine and Biology Society. 1996.

19. Ammer K. Influence of imaging and object conditions on temperature readings from medical infrared images. Polish J Environ Stud. 2006;15(4A):117-9.

20. Bohannon RW, Smith MB. Interrater reliability of a modified Ashworth scale of muscle spasticity. Phys Ther. 1987;67(2):206-7.

21. Mahoney FI, Barthel DW. Functional evaluation: the Barthel Index. Md State Med J. 1965;14:61-5.

22. Tao W, Yan D, Li JH, Shi ZH. Gait improvement by low-dose botulinum toxin A injection treatment of the lower limbs in subacute stroke patients. J Phys Ther Sci. 2015;27(3):759-62.

23. Murphy TH, Corbett D. Plasticity during stroke recovery: from synapse to behaviour. Nat Rev Neurosci. 2009;10(12):861-72.

24. Moon HI, Lee HJ, Yoon SY. Lesion location associated with balance recovery and gait velocity change after rehabilitation in stroke patients. Neuroradiology. 2017;59(6):609-18.

25. Globas C, Becker C, Cerny J, et al. Chronic stroke survivors benefit from high-intensity aerobic treadmill exercise: a randomized control trial. Neurorehabil Neural Repair. 2012;26(1):85-95.

26. Pang MY, Eng JJ. Determinants of improvement in walking capacity among individuals with chronic stroke following a multi-dimensional exercise program. J Rehabil Med. 2008;40(4):284-90.

27. Debiec-Bak A, Wojtowicz D, Pawik L, Ptak A, Skrzek A. Analysis of body surface temperatures in people with Down syndrome after general rehabilitation exercise. J Therm Anal Calorim. 2019;135(4):2399-410.

28. Drzazga Z, Binek M, Pokora I, Sadowska-Krepa E. A preliminary study on infrared thermal imaging of cross-country skiers and swimmers subjected to endurance exercise. J Therm Anal Calorim. 2018;134(1):701-10.

29. Christensen J, Matzen LH, Vaeth M, Schou S, Wenzel A. Thermography as a quantitative imaging method for assessing postoperative inflammation. Dentomaxillofac Radiol. 2012;41(6):494-9.

30. Bagavathiappan S, Philip J, Jayakumar T, et al. Correlation between plantar foot temperature and diabetic neuropathy: a case study by using an infrared thermal imaging technique. J Diabetes Sci Technol. 2010;4(6):1386-92.

31. Lim MJ, Kwon SR, Jung KH, Joo K, Park SG, Park W. Digital thermography of the fingers and toes in Raynaud's phenomenon. J Korean Med Sci. 2014;29(4):502-6.

32. Dymarek R, Taradaj J, Rosinczuk J. Extracorporeal shock wave stimulation as alternative treatment modality for wrist and fingers spasticity in poststroke patients: a prospective, open-label, preliminary clinical trial. Evid Based Complement Alternat Med. 2016;2016:4648101.

Publisher's Note Springer Nature remains neutral with regard to jurisdictional claims in published maps and institutional affiliations. 